\title{
Delimitação das fácies-reservatórios utilizando curvas de perfilagem geofísica de poços no Campo de Namorado na Bacia de Campos-RJ
}

Jomar José Knaip Ribeiro (UFES); Vitória Felício Dornelas (UFES); Carlos André Maximiano da Silva (UFES).

Copyright 2016, SBGf - Sociedade Brasileira de Geofísica

Este texto foi preparado para a apresentação no VII Simpósio Brasileiro de Geofísica, Ouro Preto, 25 a 27 de outubro de 2016. Seu conteúdo foi revisado pelo Comitê Técnico do VIISimBGf, mas não necessariamente representa a opinião da SBGf ou de seus associados. É proibida a reprodução total ou parcial deste material para propósitos comerciais sem prévia autorização da SBGf.

\section{Abstract}

Sampling campaigns are essential for the development of research and planning of an oil field. The wireline logging is an indirect sampling tool which configures extremely effective due to the reduced cost of operation and handling versatility. This study was made using acquisition date logs Gamma Ray (GR), Sonic (DT), Resistivity (ILD), Density (RHOB) and Neutron (NPHI) plotted on the LogPlot7 ${ }^{\circledR}$ software for the determination of the wells lithology and the fluids contact zones in two different wells. In both, were delineated possible reservoir rocks via the low amounts found in Gamma Rays (GR) and density (RHOB) profiles, inferring the presence of saturating fluid in the rock. It is assumed that the well 4RJS 42 has presence of oil and 3NA_004_RJS gas, based on the values generated by the resistivity profile (ILD).

\section{Introdução}

A perfilagem geofísica consiste no registro contínuo dos parâmetros geofísicos captados ao longo da parede do poço, por meio de ferramentas a cabo, ou ainda, de ferramentas acopladas à coluna de perfuração (RIDER, 2000 apud ROSA, 2006).

Qualquer que seja o tipo de perfil produzido, este não pode ser utilizado de maneira única para avaliar o potencial exploratório de um poço. $\mathrm{Na}$ realidade, para a obtenção de características confiáveis da formação de interesse é necessário a integração de diversos perfis.

Na engenharia de Petróleo, segundo Nery (2004), a aplicação dos perfis em poços abertos é encontrada nas seguintes áreas: Engenharia de Reservatório, Engenharia de Controle, Engenharia de Completação e Perfuração.

De posse das propriedades do reservatório tais como: espessura, litologia da formação, porosidade, permeabilidade, saturação da água e saturação de hidrocarbonetos, é possível predizer, a qualidade da formação quanto a acumulação de hidrocarbonetos, e a viabilidade da produção do campo. (PINHEIRO, 2014)

Os poços, 4RJS_42 e 3NA_004_RJS, estudados neste presente artigo estão localizados no Campo Escola de Namorado na Bacia de Campos RJ, Figura 1, onde foram corridos os equipamentos necessários para a obtenção de dados como raio gama, resistividade, porosidade, densidade e neutrão, para posterior analise em forma de perfis.

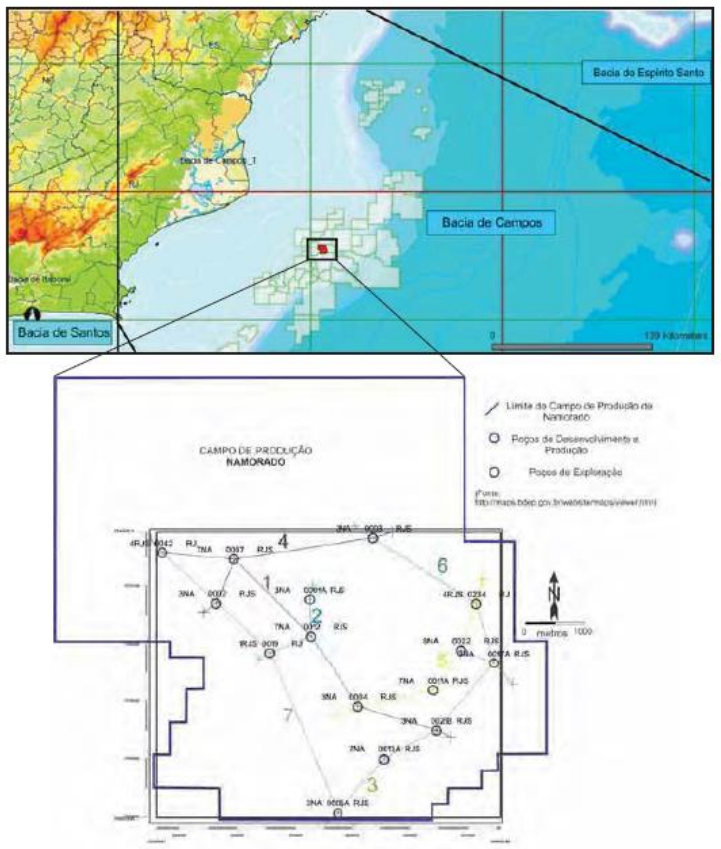

Figura 1: Localização do Campo de Namorado na Bacia de Campos RJ (BDEP/WEBMAPS).

Este trabalho visa reforçar a importância da integração dos perfis de poços no que se diz respeito a exploração de um reservatório. Foram cruzados os dados de densidade e raios gama para identificação da litologia e os dados de neutrão com densidade para obter as possíveis zonas de acumulação de óleo. Os perfis gerados serão apresentados posteriormente bem como resultados de suas análises.

\section{Metodologia}

A proposta deste estudo é de identificar as possíveis formações acumuladoras de hidrocarbonetos, nos poços 4RJS 42 e 3NA 004 RJS, localizado no campo de Namorado - Bacia de Campos, com base em dados geofísicos disponibilizados na literatura pela ANP - Agência Nacional do Petróleo. Foram utilizados dados, em formato .LAS, de perfis realizados no poços. Os dados utilizados correspondem às leituras dos perfis de raios gama (GR), sônico (DT), resistividade (ILD), densidade (RHOB) e neutrão (NPHI).

Os perfis foram gerados através da versão demo do software LogPlot7®. Este é de posse da empresa RockWare ${ }^{\circledR}$ e tanto sua licença quanto sua versão demo 
podem ser encontradas no site $<w w w$.rockware.com>. A versão demo possui duração de dez dias e somente vinte e cinco novos acessos podem ser feitos.

O perfil de raios gama (GR) permite identificar o índice de raios gama, com isso o volume de argila na áreaperfilada e por consequência sua litologia. Quando associado ao perfil de densidade (RHOB) permite identificar o tipo do reservatório.

O índice de argilosidade é dado em API por:

$\mathrm{I}_{\mathrm{GR}}=V_{S H}=\left(G R_{\text {lido }}-G R_{\text {minimo }}\right) /\left(G R_{\text {maximo }}-G R_{\text {minimo }}\right)$

Os Perfis sônicos (DT )são obtidos pelo tempo que um pulso sonoro leva para atravessar determinado intervalo de formação geológica. Relaciona diretamente tempo de trânsito e porosidade da formação rochosa, ou seja: quanto maior o tempo de trânsito, menor a densidade da formação. Tempo de transito é dado em $\mu \mathrm{s} / \mathrm{ft}$ e a porosidade Sônica é calculada em \% pela fórmula:

$\Phi_{\mathrm{t}}=\left(\Delta t_{\text {lido }}-\Delta t_{\text {matriz }}\right) /\left(\Delta t_{\text {fluido }}-\Delta t_{\text {matriz }}\right)$

O perfil de resistividade (ILD) é o registro em profundidade, das variações da condutividade das rochas, medidas na região não perturbada pelos efeitos da invasão. Sendo normalmente expresso em unidades de resistividade, ohm.m (SILVA, 2011), a Figura 2 apresenta os valores de resistividade adotados comumente para os fluidos. O perfil de resistividade (ILD) indica quais áreas possuem maior probabilidade de conter óleo, através da resistividade lida na região de interesse.

\begin{tabular}{ccc} 
Água salgada & Água doce & Óleo \\
\hline 1 & $\mid$ & $\mid$ \\
1,0 & 1,5 & 10,0
\end{tabular}

Figura 2: Faixa de resistividade (em $\Omega . \mathrm{m})$ da água salgada, água doce e óleo.

O perfil de Densidade (RHOB) utiliza da detecção de raios gama desviada pelos sedimentos para medir a densidade das rochas. Esse perfil é útil para estimar a porosidade e densidade das camadas, facilitando identificar zonas com gás (CARVALHO, 2014). A densidade é obtida em $\mathrm{g} / \mathrm{cm}^{3}$ e é possível calcular a porosidade-densidade,em \%, por:

$\Phi_{\rho}=\left(\rho_{\text {matriz }}-\rho_{\text {lido }}\right) /\left(\rho_{\text {matriz }}-\rho_{\text {fluido }}\right)$

O perfil neutrônico (NPHI)registra o índice de hidrogênio presente nas camadas sedimentares, já que esse elemento está contido nas moléculas dos fluidos que preenchem os poros das rochas, a exemplo da água e dos hidrocarbonetos (GONZALEZ, 2014).

\section{Resultados}

Inicialmente foi criado o design dos perfis no software LogPlot7® (Figura 3). Nessa etapa, foram escolhidos os cinco perfis supracitados com as características referentes a estes (nome do perfil, valor mínimo e máximo, cor da curva, escala, características do grid). Os perfis de Densidade e Neutrão foram aglutinados em uma mesma faixa (track) a fim de que a leitura seja facilitada com esta configuração.

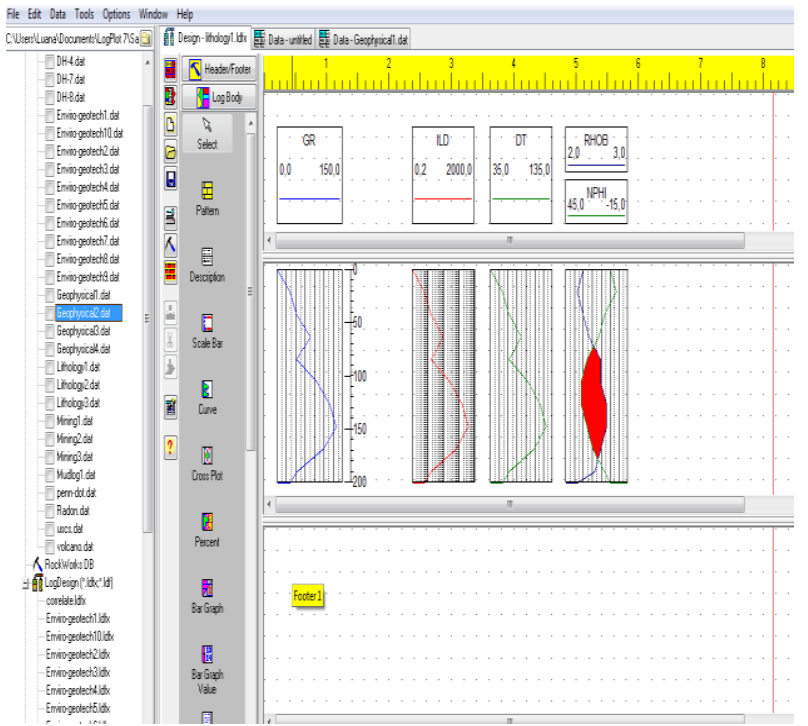

Figura 3: Design dos perfis no software LogPlot7®.

Posteriormente, os dados no formato .LAS foram convertidos para o Excel, com valores respectivos de cada perfil, e importados para o software (Figura 4). Por fim, clicando no ícone de compilar perfis, foi possível formar o conjunto de perfis encontrados na Figura 5.

\begin{tabular}{|c|c|c|c|c|c|c|}
\hline & \multicolumn{2}{|c|}{\begin{tabular}{|l|l|l|l} 
SETUP & E-TEXT E
\end{tabular}} & \multirow[b]{2}{*}{ IILD } & \multirow[b]{2}{*}{ DT } & \multirow[b]{2}{*}{ RHOB } & \multirow[b]{2}{*}{ NPHI } \\
\hline & Depth & GR & & & & \\
\hline $2^{2}$ & 2975 & 66,4531 & 1,8425 & 91,2695 & 2,4617 & 21,7996 \\
\hline 묘 & 2975 & 66,4531 & 1,8425 & 91,2695 & 2,4617 & 21,7996 \\
\hline 品 & 2975,2 & 68,4648 & 1,7627 & 90,9399 & 2,4266 & 21,8086 \\
\hline$\therefore$ & 2975,4 & 69,0938 & 1,7114 & 90,3281 & 2,427 & 23,1367 \\
\hline$B$ & 2975,6 & 67,7969 & 1,6621 & 87,7031 & 2,4411 & 24,6211 \\
\hline R & 2975,8 & 67,1992 & 1,594 & 85,2031 & 2,4502 & 25,5234 \\
\hline 置 & 2976 & 67,957 & 1,5305 & 86,5234 & 2,4579 & 26,3086 \\
\hline z & 2976,2 & 69,0273 & 1,4912 & 91,1875 & 2,4529 & 26,2773 \\
\hline 连 & 2976,4 & 69,875 & 1,4514 & 94,457 & 2,4197 & 25,7148 \\
\hline$?$ & 2976,6 & 70,5117 & 1,4237 & 95,6133 & 2,3845 & 25,1562 \\
\hline & 2976,8 & 70,3398 & 1,4192 & 97,0078 & 2,3754 & 24,865 \\
\hline & 2977 & 68,6406 & 1,4285 & 97,0391 & 2,3744 & 25,0002 \\
\hline & 2977,2 & 66,5039 & 1,4381 & 96,2578 & 2,3668 & 25,1091 \\
\hline & 2977,4 & 65,207 & 1,44 & 96,0483 & 2,3782 & 25,0427 \\
\hline & 2977,6 & 64,8555 & 1,4288 & 95,9258 & 2,3969 & 25,125 \\
\hline & 2977,8 & 65,7578 & 1,4145 & 95,5117 & 2,4038 & 25,5703 \\
\hline & 2978 & 67,1523 & 1,3992 & 94,957 & 2,4061 & 26,3633 \\
\hline & 2978,2 & 67,2812 & 1,3757 & 94,6917 & 2,4109 & 27,6094 \\
\hline & 2978,4 & 67,2734 & 1,3442 & 95,4727 & 2,4249 & 29,0703 \\
\hline & 2978,6 & 68,2148 & 1,3255 & 96,2383 & 2,4276 & 30,1758 \\
\hline & 2978,8 & 68,6182 & 1,3254 & 96 & 2,4191 & 29,9648 \\
\hline
\end{tabular}

Figura 4: Dados dos perfis importados para o software LogPlot7®. 

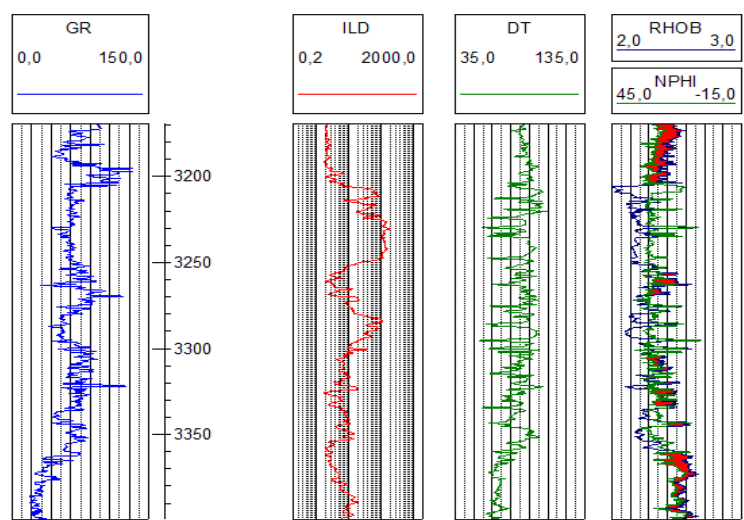

Figura 5: Perfis do poço visualizados no LogPlot7 $7^{\circledR}$.

Nessa fase, segundo Nery (2004), foi realizada a integração das análises dos perfis como forma de assegurar maior precisão dos resultados. Com o objetivo de tirar informações da suíte básica de perfis através do cálculo e estimação de outras curvas, o fluxo de trabalho se mostrou eficiente, correspondendo com a realidade $\mathrm{e}$ mostrando semelhança com trabalhos anteriores sobre o Campo de Namorado.

Para iniciar o processo de avaliação, foi necessário definir os principais litotipos na região. De acordo com Silva, Portugal \& Vidal (2010), no campo de Namorados, estes foram agrupados em três eletrofácies por meio da combinação dos perfis GR e RHOB:

1) Arenito reservatório: identificado por baixos valores de GR e RHOB.

2) Sedimentos finos: identificado por altos valores de GR e RHOB.

3) Calcário: identificado por baixos valores de GR e altos valores de RHOB.

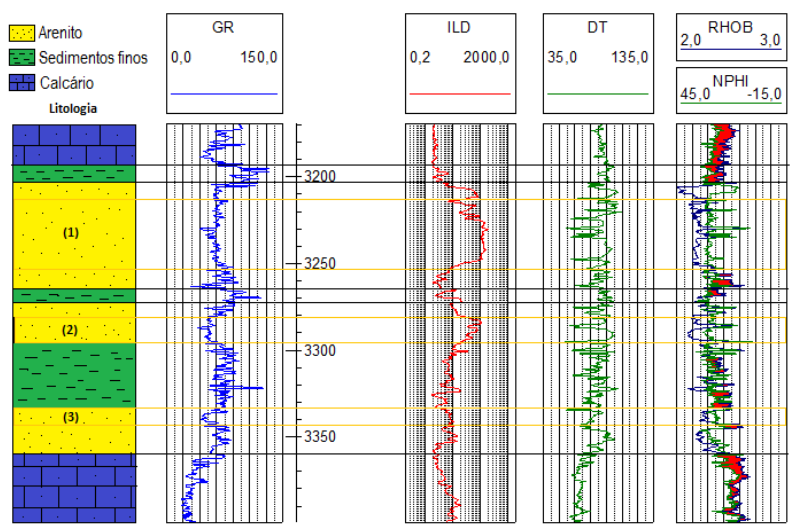

Figura 6: Perfis do Poço 4RJS_42 com delimitação das prováveis fácies-reservatórios areníticas. Destacando as regiões onde é esperado que haja contenção de fluidos.

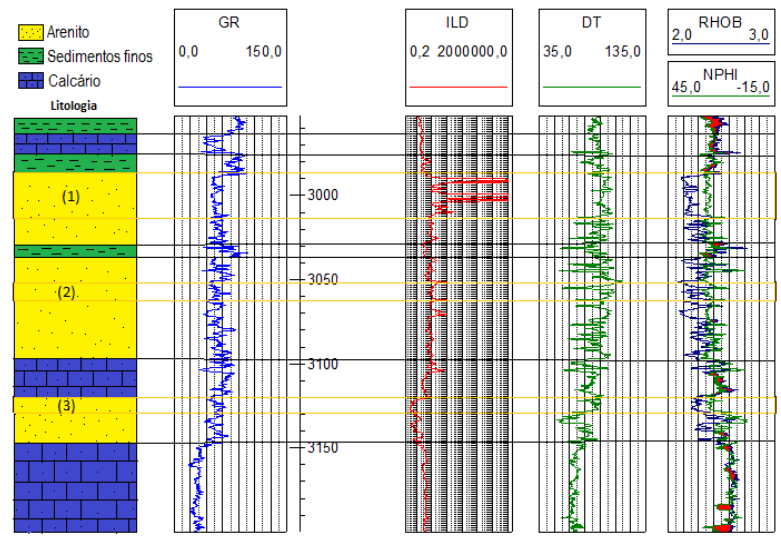

Figura 7:Perfis do Poço 3NA_004_RJS com delimitação das prováveis fácies-reservatórios areníticas. Destacando as regiões onde é esperado que haja contenção de fluidos.

\section{Discussão e Conclusões}

Através das técnicas de interpretação de perfis de poços, aplicada aos dados relativos aos poços 4RJS_42 e 3NA 004_RJS, plotados com auxílio do software LogPlot $7^{\mathbb{\Theta}}$, o presente trabalho caracterizou as possíveis rochas reservatório e as zonas de contato entre os fluidos.

Em ambos poços analisados foram identificados três zonas de reservatório arenítico com potencial para conter óleo. Analisando o perfil de resistividade e tomando os valores para identificação de fluidos da Figura 6, podemos dizer que as zonas 1 e 2 do poço 4RJS 42 tem potencial reservatório de óleo. Assim como a região 1 do poço 3NA_004_RJS (Figura 7) possui potencial de contenção de gás, isto é observado atrás da alta resistividade da região e através das curvas de densidade e neutrão que apresentam comportamento característico de presença de gás.

Contudo é necessário ressaltar que somente análises mais profundas do reservatório, tais como testemunhagens e análise PVT, permitirão um maior conhecimento sobre o real conteúdo do mesmo. E apenas a perfuração realmente comprovará a existência de fluidos no mesmo.

A perfilagem é um dos primeiros métodos de análise, e muitas vezes ela carece de uma maior quantidade de dados, que podem não estar disponíveis no momento. Por exemplo, área reservatório que é dependente da sísmica ou densidades mais específicas e condizentes com as formações e fluidos pelo qual o poço atravessa que só se comprovam após a perfuração e análises laboratoriais. 


\section{Agradecimentos}

dados.

Os autores agradecem à ANP pela liberação dos

\section{Referências}

BDEP. Produção de Petróleo e Gás Natural por campo. Rio de Janeiro, RJ, Disponível em:<http://www.bdep.gov.br/?id=441>. Acesso em: $08 / 01 / 2016$

CARVALHO, P. B. Caraterização petrofísica do campo de namorado a partir de perfis de poços. Graduação em Geofísica. Universidade Federal Fluminense. 2014.

GONZALEZ, S. P. Estimativa de saturação de água conata em reservatórios clásticos. Universidade Federal Fluminense, 2014.

LOGPLOT 7® - DEMO. Disponível em: <www.rockware.com>. Acesso em: 02/02/2016.

NERY, G. G. Perfilagem Geofísica em Poço Aberto. Salvador - BA, 2004.

PINHEIRO, L.N.P. Caracterização do reservatório Carapebus do campo de Peregrino, bacia de Campos, através da análise de perfis geofísicos de poços, integrada à interpretação sísmica. Graduação em Geofísica. Universidade Federal Fluminense.2014.

ROSA, Henrique. Estudo de caracterização de eletrofácies por meio de perfis geofísicos de poços e de amostras de testemunhos utilizando estatística multivariada. 2006. 230 f. Dissertação (Doutorado) Universidade Estadual de Campinas, Campinas, 2006.

SILVA, C. B. Solução da Equação de Archie com Algoritmos Inteligentes. 2011. 82 f. Dissertação (Doutorado) - Universidade Federal do Pará, Belém, 2011.

SILVA, E. P. A.; PORTUGAL, R. de S.; VIDAL, A. C. Modelagem AVO - Estudo de caso em um poço no campo de Namorado. Revista Brasileira de Geofísica. Campinas. São Paulo. 2010. 